\title{
Radiation Therapy for Patients with Bone Metastasis from Uterine Cervical Cancer: Its Role and Optimal Radiation Regimen for Palliative Care
}

\author{
MASAHARU HATA ${ }^{1,2}$, IZUMI KOIKE ${ }^{2}$, ETSUKO MIYAGI ${ }^{3}$, MIKIKO ASAI-SATO ${ }^{3}$, HISASHI KAIZU ${ }^{2}$,

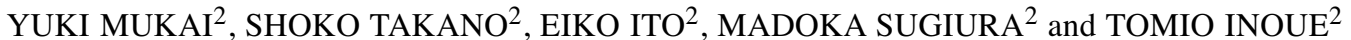 \\ ${ }^{1}$ Division of Radiation Oncology, Department of Oncology, \\ Yokohama City University Graduate School of Medicine, Yokohama, Japan; \\ Departments of ${ }^{2}$ Radiology, and ${ }^{3}$ Obstetrics and Gynecology, \\ Yokohama City University Graduate School of Medicine, Yokohama, Japan
}

\begin{abstract}
Aim: To determine the role of radiation therapy for patients with bone metastasis from uterine cervical cancer and identify an optimal radiation regimen. Patients and Methods: A total of 20 patients with bone metastases from uterine cervical cancer received radiation therapy to the pelvis. The median total dose of $60.2 \mathrm{~Gy}$ in the $2 \mathrm{~Gy}$ per fraction-equivalent dose (EQD2) was delivered to cervical tumors of all patients. Thirteen patients underwent chemotherapy during and/or following radiation therapy. Results: In 18 of 20 patients, the primary tumors disappeared or were markedly reduced after radiation therapy. In all but one of 17 patients with pelvic pain and bleeding, these symptoms disappeared or were remarkably relieved. However, three patients had primary tumor progression at 7, 9, and 15 months after irradiation with total doses of 56.8, 58.4, and 68.3 Gy in EQD2, respectively. Two of these patients had relapses of bleeding and pain. The primary progression-free rate considering all patients was $69 \%$ at 1 year and 34\% at 2 years. The corresponding overall survival rates were $34 \%$ at 1 year, and $8 \%$ at 2 years, with an estimated median survival time of 7 months. The number of metastatic bone sites $(p=0.027)$ and administration of chemotherapy $(p<0.001)$ were significant prognostic factors for survival. Conclusion: Radiation therapy is effective for relief of pelvic symptoms in patients
\end{abstract}

Correspondence to: Masaharu Hata, MD, Division of Radiation Oncology, Department of Oncology, Yokohama City University Graduate School of Medicine, 3-9 Fukuura, Kanazawa-ku, Yokohama, Kanagawa 236-0004, Japan. Tel: +81 457872696, Fax: +81 457860369, e-mail: mhata@syd.odn.ne.jp

Key Words: Bone metastasis, cervical cancer, palliative care, radiation therapy, uterine cervix. with bone metastasis from uterine cervical cancer. For patients who are expected to survive for more than 1 year, almost curative-dose irradiation to primary tumors is recommended.

Uterine cervical cancer is one of the major malignancies and the fourth most common after breast, colorectal, and lung cancer in women worldwide. Annually 528,000 women are newly diagnosed with uterine cervical cancer and 266,000 die of this disease, accounting for $7.5 \%$ of all female deaths from cancer $(1,2)$. Many cases of uterine cervical cancer (about $85 \%$ ) are found in developing countries, where it is the most common malignancy in women and accounts for almost $12 \%$ of all female cancer. In developed countries, the spread of screening for uterine cervical cancer and vaccination against human papillomavirus have contributed to detecting the disease at an earlier stage and reducing its incidence, respectively. However, some patients are still found to have advanced-stage disease at diagnosis. In fact, around $3 \%$ of patients at diagnosis have uterine cervical cancer at stage IVB with spread of the tumor beyond the pelvis, such as lung, liver, and bone metastases, based on the International Federation of Gynecology and Obstetrics staging system $(3,4)$.

Patients with distant metastasis in the visceral organs from uterine cervical cancer are usually incurable and are candidates for palliative care $(5,6)$. Patients with bone metastasis are particularly likely to have a relatively poor prognosis. Even in such patients, radiation therapy has been positively used with palliative intent to relieve severe symptoms, including bleeding and pain, via primary tumor control. However, few studies have analyzed patient data in detail, and the most appropriate radiation regimen for patients with bone metastasis from uterine cervical cancer remains unclear. In our previously published study, we reported treatment outcomes of patients 
with distant metastasis from uterine cervical cancer treated with radiation therapy, but were unable to draw pertinent conclusions because of the small number of patients with bone metastasis (7). We therefore updated and re-analyzed the patient data to determine the role of radiation therapy for patients with bone metastasis from uterine cervical cancer and to identify the optimal radiation regimen.

\section{Patients and Methods}

Patients. Between July 2001 and November 2016, a total of 20 patients with bone metastasis from uterine cervical cancer received radiation therapy to the pelvis at our Institution. All patients had pathologically confirmed uterine cervical carcinoma. Histology was squamous cell carcinoma in 11 patients, adenocarcinoma in four, adenosquamous carcinoma in one, and neuroendocrine carcinoma in one. The remaining three patients had such poorly differentiated carcinomas that it was impossible to make a definitive diagnosis, such as squamous cell carcinoma or adenocarcinoma. Clinical stage was assessed by chest X-ray and computed tomography (CT), abdominal CT, pelvic CT and magnetic resonance imaging, bone scintigraphy, and/or ${ }^{18} \mathrm{~F}$-fluorodeoxyglucose positron-emission tomography prior to radiation therapy. Bladder and rectal invasion were ascertained by cystoscopy and colonoscopy, respectively. Of the 20 patients, 16 had pelvic lymph node metastases. Lymph node enlargement $>10 \mathrm{~mm}$ in the short axis on CT was defined as metastasis. All patients had bone metastases (solitary bone in five patients and multiple bones in 15), and 17 patients also had distant metastases in other sites, such as lung, liver, and lymph nodes in the distant areas including the supraclavicular and para-aortic regions. Consequently, all patients were clinically diagnosed as having stage IVB disease with bone metastasis, based on the TNM classification defined by the Union for International Cancer Control (8).

Patient characteristics are shown in Table I. Informed consent was obtained from all patients before treatment.

Radiation therapy. All 20 patients initially received external irradiation to the whole pelvis in antero-posterior opposed fields or antero-posterior and bilateral (box) fields with 14- or 15-MV Xrays. Patients received 1.8-2.5 Gy (median=1.8 Gy) per day, five times per week, to total doses of 32.4-52.2 Gy (median=50.4 Gy) in 18-29 fractions (median, 28). Twelve out of the 20 patients also received high-dose-rate (HDR) intracavitary brachytherapy along with external irradiation to treat the primary cervical tumors. Total doses of 6-25 Gy (median=13 Gy) in 1-5 (median=3) once-weekly fractions of 5-6 Gy (median=5 Gy) were delivered to point A using an HDR Ir-192 source (9). In the remaining eight patients, intracavitary brachytherapy was abandoned because of patient refusal, poor general condition, or technical difficulties. In four out of these eight patients, the primary tumors were treated with an external radiation boost to total doses of 54-59.4 Gy in 30-33 fractions. The total doses administered to all 20 patients were thus 32.4-76.2 Gy (median=59.9 Gy), and the overall treatment time was 20-65 days (median $=48$ days). The biological effective dose (BED) was calculated from the total physical dose according to the linear quadratic model using $\alpha / \beta$ ratios of 10 and 3 for early- and late-responding tissues, respectively; the median $\mathrm{BED}_{10}$ and $\mathrm{BED}_{3}$ were 72.3 Gy (range=38.2-100.0 Gy) and 103.0 Gy (range=51.8$155.5 \mathrm{~Gy}$ ), respectively (10). The $\mathrm{BED}_{10}$ was also considered as
Table I. Patient and tumor characteristics.

\begin{tabular}{|c|c|}
\hline Number of patients & 20 \\
\hline \multicolumn{2}{|l|}{ Age, years } \\
\hline Median (range) & $56(27-87)$ \\
\hline \multicolumn{2}{|l|}{ ECOG PS } \\
\hline 0 & 4 \\
\hline 1 & 8 \\
\hline 2 & 4 \\
\hline 3 & 3 \\
\hline 4 & 1 \\
\hline \multicolumn{2}{|l|}{ Histology } \\
\hline Squamous cell carcinoma & 11 \\
\hline Adenocarcinoma & 4 \\
\hline Adenosquamous carcinoma & 1 \\
\hline Neuroendocrine carcinoma & 1 \\
\hline Poorly differentiated carcinoma & 3 \\
\hline \multicolumn{2}{|l|}{ Maximum primary tumor diameter, $\mathrm{mm}$} \\
\hline Median (range) & $80(35-135)$ \\
\hline \multicolumn{2}{|l|}{ T Category } \\
\hline $\mathrm{T} 1 \mathrm{~b}$ & 1 \\
\hline $\mathrm{T} 2 \mathrm{a}$ & 1 \\
\hline $\mathrm{T} 2 \mathrm{~b}$ & 1 \\
\hline $\mathrm{T} 3 \mathrm{~b}$ & 7 \\
\hline $\mathrm{T} 4$ & 10 \\
\hline \multicolumn{2}{|l|}{ N Category } \\
\hline No & 4 \\
\hline N1 & 16 \\
\hline \multicolumn{2}{|l|}{ Hydronephrosis } \\
\hline Yes & 11 \\
\hline No & 9 \\
\hline \multicolumn{2}{|l|}{ No. of metastatic bone sites } \\
\hline 1 & 5 \\
\hline 2 & 5 \\
\hline 3 & 4 \\
\hline$\geq 4$ & 6 \\
\hline \multicolumn{2}{|l|}{ No. of distant metastatic organs except bone } \\
\hline 0 & 3 \\
\hline 1 & 8 \\
\hline 2 & 4 \\
\hline$\geq 3$ & 5 \\
\hline \multicolumn{2}{|l|}{ Pretreatment hemoglobin, $\mathrm{g} / \mathrm{dl}$} \\
\hline Median (range) & $9.6(5.9-12.6)$ \\
\hline \multicolumn{2}{|l|}{ Total radiation dose, EQD2, Gy } \\
\hline Median (range) & $60.2(31.9-83.3)$ \\
\hline \multicolumn{2}{|l|}{ Chemotherapy } \\
\hline Concurrent CRT alone & 1 \\
\hline Concurrent CRT followed by systemic chemotherapy & 10 \\
\hline Systemic chemotherapy after RT alone & 2 \\
\hline No chemotherapy & 7 \\
\hline
\end{tabular}

CRT, Chemoradiotherapy; ECOG PS, Eastern Cooperative Oncology Group performance status; EQD2, 2 Gy per fraction-equivalent dose; $\mathrm{RT}$, radiation therapy.

the dose of radiation that was effective for tumor treatment, and the median dose of 72.3 Gy (range=38.2-100.0 Gy) corresponded to $60.2 \mathrm{~Gy}$ (range=31.9-83.3 Gy) in the 2 Gy per fractionequivalent (conventional fraction) dose (EQD2).

Thirteen out of the 20 patients also received chemotherapy, with most receiving concurrent intravenous cisplatin $\left(40 \mathrm{mg} / \mathrm{m}^{2}\right)$ weekly 


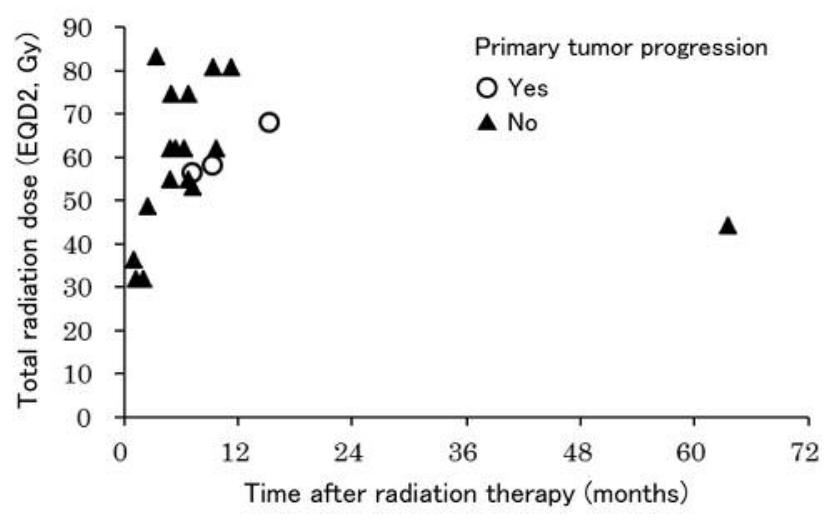

Figure 1. Relationship between total radiation dose and time after radiation therapy resulting in control of primary tumors. Three patients treated with total doses of 56.8, 58.4, and 68.3 Gy [in 2 Gy per fractionequivalent dose (EQD2)] showed primary tumor progression at 7, 9, and 15 months, respectively, after radiation therapy. during radiation therapy. In addition, systemic chemotherapy of various courses with mainly paclitaxel and cisplatin was administered to 12 patients to control distant metastasis after radiation therapy. For the remaining seven patients, chemotherapy was unusable because of renal dysfunction, poor general condition, or old age.

Follow-up and evaluation criteria. Patients were examined by pelvic CT within 1 month after completion of irradiation, and had subsequent follow-up CT scans at 3- to 12-month intervals. Patients with no regrowth of the primary tumors were considered primary progression-free.

Acute and late toxicities associated with radiation therapy were evaluated using the Radiation Therapy Oncology Group (RTOG) acute radiation morbidity scoring criteria and the RTOG/European Organization for Research and Treatment of Cancer late radiation morbidity scoring scheme, respectively (11). Acute toxicities were defined as radiation-induced toxicities occurring within 3 months after initiation of radiation therapy, and late toxicities as those occurring after 3 months.

Statistical analysis. Actuarial survival and disease control rates were calculated from the initiation of radiation therapy, according to the Kaplan-Meier method (12). The log-rank test was applied to detect probable prognostic factors considered predictable among patient and tumor factors in univariate analyses. A $p$-value of less than 0.05 was considered as being statistically significant. All statistical analyses were performed using the statistical software IBM SPSS version 22 (IBM, Armonk, NY, USA).

\section{Results}

Tumor control. In 18 out of 20 patients, the primary tumors disappeared or were markedly reduced as initial responses after treatment, and the remaining two showed no change; the objective response rate was therefore $90 \%$. However, three patients with T3b-T4 tumors had primary tumor

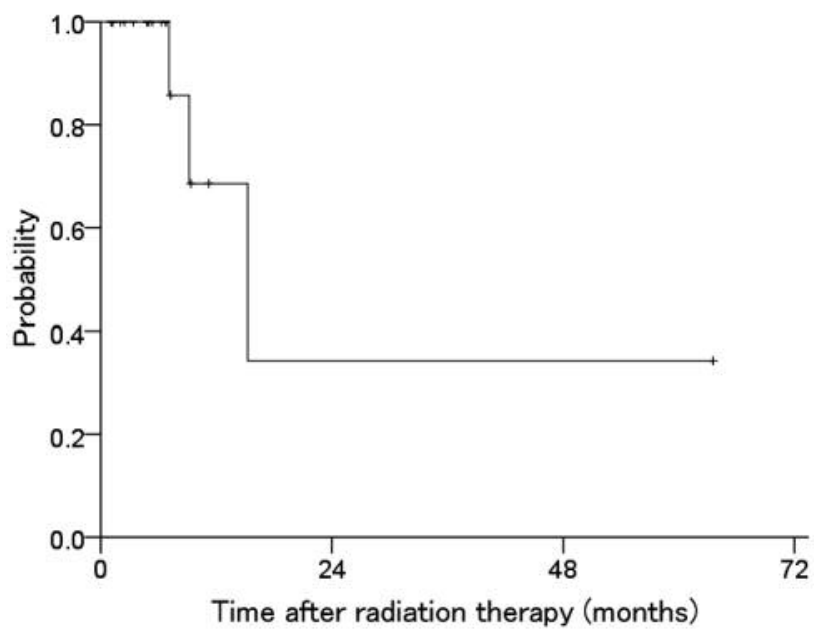

Figure 2. Primary tumor control in 20 patients with bone metastasis from uterine cervical cancer treated with radiation therapy.

progression at 7, 9, and 15 months after radiation therapy, and histology showed squamous cell carcinoma in the former two patients and adenocarcinoma in the latter. The $\mathrm{BED}_{10}$ of these three patients with primary tumor progression was $68.1,70.1$, and $82.0 \mathrm{~Gy}$, corresponding to $56.8,58.4$, and 68.3 Gy in EQD2, respectively (Figure 1). All but two of the 20 patients had progression of distant metastases. The remaining two patients showed neither progression of primary tumors nor distant metastases at 9 and 64 months after concurrent chemoradiotherapy followed by systemic chemotherapy. The latter patient had distant metastases in the lung and mediastinal lymph node, besides the bone, from poorly differentiated carcinoma and received external pelvic irradiation at a total dose of $45 \mathrm{~Gy}$ in 25 fractions. After treatment, this patient achieved complete response in the bulky primary tumor of T2b (103 $\mathrm{mm}$ in maximum diameter) and all distant metastases, and then showed no recurrence over a longer term of more than 5 years.

The primary progression-free rate considering all patients was $69 \%$ at 1 year and $34 \%$ at 2 years (Figure 2).

Relief of symptoms. In all but one out of 17 patients with bleeding from the primary cervical tumor before radiation therapy, bleeding remarkably decreased following treatment, with an objective hemostatic rate of $94 \%$. In particular, 14 patients had complete hemostasis. Concerning pelvic pain, radiation therapy was also very effective. All 12 patients with pelvic pain derived from the tumors before radiation therapy had significant pain relief after treatment, with 10 being completely pain-free. However, of the three patients with primary tumor progression, two had relapses of bleeding and pain. These two patients suffered from progressive pain and 


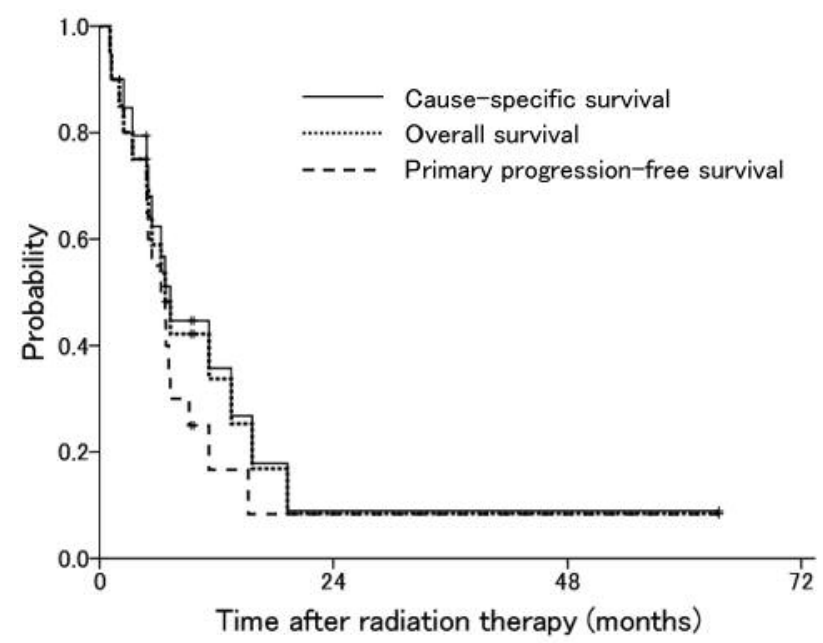

Figure 3. Survival in 20 patients with bone metastasis from uterine cervical cancer treated with radiation therapy.

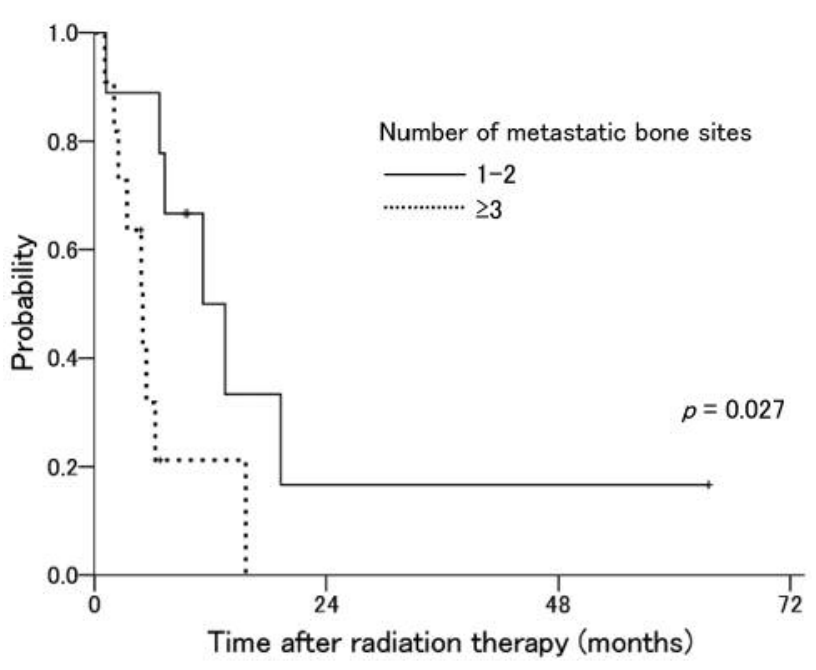

Figure 4. Overall survival in 20 patients with bone metastasis from uterine cervical cancer treated with radiation therapy according to the number of metastatic bone sites. Patients with 1-2 metastatic bone sites showed significantly better survival than patients with 3 or more metastatic bone sites $(p=0.027)$.

needed a transfusion of red blood cells that corresponded to 1.3 and 2.91 of blood in total because of bleeding from their progressive tumors during 6 and 10 months, respectively, between primary tumor progression and death. The remaining patient died of disease immediately after the emergence of primary tumor progression.

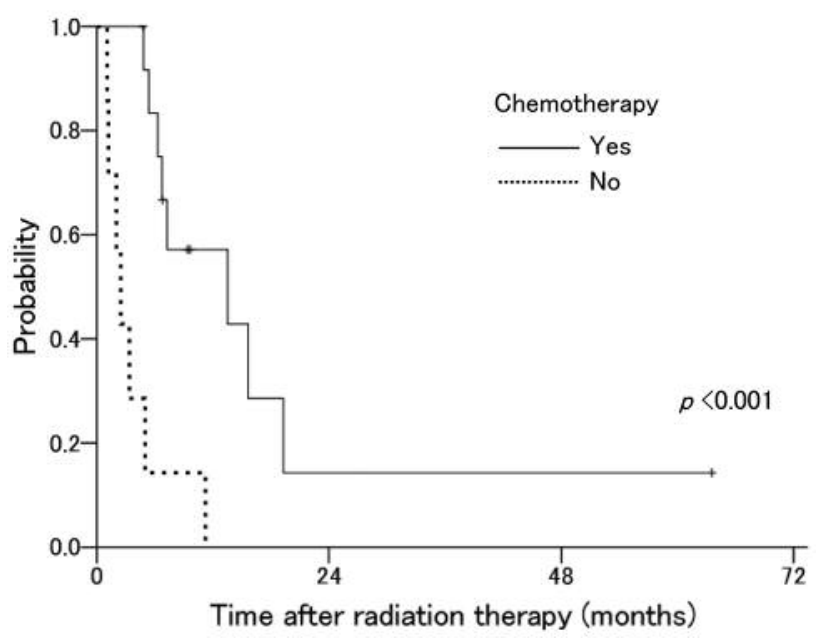

Figure 5. Overall survival in 20 patients with bone metastasis from uterine cervical cancer treated with radiation therapy according to chemotherapy. Patients who received chemotherapy displayed significantly improved survival compared to patients who received no chemotherapy $(p<0.001)$.

Survival. Fifteen out of the 20 patients, including three with primary tumor progression, died at 1-19 months (median, 5 months) after radiation therapy. Of these 15 patients, 14 died of disease progression and one died of sepsis from a cancerunrelated cause. There was no mortality associated with treatment. The remaining five patients survived, with no primary tumor progression at the last follow-up of 5-64 months (median $=9$ months) after radiation therapy. As a result, only four and one patient survived longer than 1 and 2 years, respectively.

The primary progression-free, cause-specific, and overall survival rates considering all patients were $17 \%, 36 \%$, and $34 \%$ at 1 year, and $8 \%, 9 \%$, and $8 \%$ at 2 years, respectively, with an estimated median survival time of 7 months (Figure 3).

Evaluation of prognostic factors. As shown in Table II, the number of metastatic bone sites $(p=0.027)$ and chemotherapy $(p<0.001)$ were considered as significant variables affecting overall survival. The 1 -year overall survival rates were $50 \%$ and $21 \%$ in patients with distant metastasis in 1-2 and $\geq 3$ bone sites, respectively, demonstrating a significant difference (Figure 4). Patients treated with chemotherapy showed an evidently better 1year overall survival rate of $57 \%$, compared with $0 \%$ in those not treated with chemotherapy (Figure 5). Both the patients with primary tumor progression and relapse of bleeding and pain bore these two favorable prognostic factors.

On the other hand, there were no significant prognostic factors for primary tumor control. Although none of the 
Table II. Results of Kaplan-Meier and univariate analyses of prognostic factors regarding primary tumor control and survival at 1 year.

\begin{tabular}{|c|c|c|c|c|c|}
\hline Prognostic factor & No. of patients & Primary tumor control (\%) & $p$-Value & Overall survival (\%) & $p$-Value \\
\hline \multicolumn{6}{|l|}{ Age, years } \\
\hline$<56$ & 10 & 53 & \multirow[t]{2}{*}{0.824} & 57 & \multirow[t]{2}{*}{0.122} \\
\hline$\geq 56$ & 10 & 100 & & 15 & \\
\hline \multicolumn{6}{|l|}{ ECOG PS } \\
\hline $0-1$ & 12 & 100 & \multirow[t]{2}{*}{0.140} & 31 & \multirow[t]{2}{*}{0.486} \\
\hline $2-4$ & 8 & 38 (at 9 months) & & 38 & \\
\hline \multicolumn{6}{|l|}{ Histology } \\
\hline Squamous cell carcinoma & 11 & 50 (at 11 months) & \multirow[t]{2}{*}{0.234} & 34 & \multirow[t]{2}{*}{0.968} \\
\hline Others & 9 & 100 & & 30 & \\
\hline \multicolumn{6}{|c|}{ Maximum primary tumor diameter, $\mathrm{mm}$} \\
\hline$<80$ & 10 & 60 & \multirow[t]{2}{*}{0.208} & 53 & \multirow[t]{2}{*}{0.212} \\
\hline$\geq 80$ & 10 & 100 & & 12 & \\
\hline \multicolumn{6}{|l|}{ T Category } \\
\hline T1-T3 & 10 & 75 & \multirow[t]{2}{*}{0.433} & 46 & \multirow[t]{2}{*}{0.140} \\
\hline $\mathrm{T} 4$ & 10 & 50 & & 20 & \\
\hline \multicolumn{6}{|l|}{ N Category } \\
\hline N0 & 4 & 100 & \multirow[t]{2}{*}{0.702} & 25 & \multirow[t]{2}{*}{0.988} \\
\hline N1 & 16 & 50 & & 41 & \\
\hline \multicolumn{6}{|l|}{ Hydronephrosis } \\
\hline Yes & 11 & 50 & \multirow[t]{2}{*}{0.433} & 21 & \multirow[t]{2}{*}{0.174} \\
\hline No & 9 & 75 & & 44 & \\
\hline \multicolumn{6}{|l|}{ No. of metastatic bone sites } \\
\hline $1-2$ & 9 & 63 & \multirow[t]{2}{*}{0.829} & 50 & \multirow[t]{2}{*}{0.027} \\
\hline$\geq 3$ & 11 & 100 & & 21 & \\
\hline \multicolumn{6}{|c|}{ No. of metastatic visceral organs except bone } \\
\hline $0-1$ & 11 & 60 & \multirow[t]{2}{*}{0.208} & 41 & \multirow[t]{2}{*}{0.883} \\
\hline$\geq 2$ & 9 & 100 & & 19 & \\
\hline \multicolumn{6}{|l|}{ Pretreatment hemoglobin, g/dl } \\
\hline$<9.6$ & 9 & 53 & 0.824 & 53 & 0.178 \\
\hline$\geq 9.6$ & 11 & 100 & & 16 & \\
\hline Total radiation dose, EQD2, & & & & & \\
\hline$<60.2$ & 10 & 38 & 0.538 & 30 & 0.550 \\
\hline$\geq 60.2$ & 10 & 100 & & 28 & \\
\hline Chemotherapy & & & & & \\
\hline Yes & 13 & 63 & 0.519 & 57 & $<0.001$ \\
\hline No & 7 & 100 (at 11 months) & & 0 & \\
\hline
\end{tabular}

ECOG PS, Eastern Cooperative Oncology Group performance status; EQD2, 2 Gy per fraction-equivalent dose.

patients treated with doses of 72.3 Gy or higher in the $\mathrm{BED}_{10}$ developed primary tumor progression within 1 year, the total radiation dose was not statistically significant for primary tumor control.

Toxicity. There were no acute grade 3 or higher toxicities except for transient hematological toxicities (Table III). Grade 3 or more leukopenia and anemia developed in three and four patients, respectively. All three patients with grade 3 leukopenia were treated with concurrent chemoradiotherapy.

No patient developed grade 2 or more therapy-related late toxicities after treatment.

\section{Discussion}

Generally, patients with disease at stage IVB with distant metastasis from uterine cervical cancer, with the exception of isolated para-aortic lymph node metastasis, are considered to be incurable and have a poor prognosis $(4,13)$. For these patients, the 5-year overall survival rate is less than $10 \%$ and the median survival time is almost 1 year. While chemotherapy undoubtedly contributes to the improvement of patient prognosis, the prolonged survival time is only a few months $(14,15)$. Specifically, bone metastasis is one of the most unfavorable prognostic factors undermining patient survival. For these patients, best supportive care is usually 
Table III. Number of patients with therapy-related acute toxicities according to the acute radiation morbidity scoring criteria of the Radiation Therapy Oncology Group (11).

\begin{tabular}{|c|c|c|c|c|}
\hline \multirow[t]{2}{*}{ Toxicity } & \multicolumn{4}{|c|}{ Grade } \\
\hline & 1 & 2 & 3 & 4 \\
\hline \multicolumn{5}{|l|}{ Hematological } \\
\hline Leukopenia & 3 & 6 & 3 & 0 \\
\hline Anemia & 4 & 12 & 3 & 1 \\
\hline Thrombocytopenia & 1 & 0 & 0 & 0 \\
\hline \multicolumn{5}{|l|}{ Skin } \\
\hline $\begin{array}{l}\text { Erythema/moist } \\
\text { desquamation }\end{array}$ & 3 & 4 & 0 & 0 \\
\hline \multicolumn{5}{|l|}{ Upper GI } \\
\hline $\begin{array}{l}\text { Abdominal discomfort/ } \\
\text { nausea/vomiting }\end{array}$ & 12 & 2 & 0 & 0 \\
\hline \multicolumn{5}{|l|}{ Lower GI } \\
\hline Diarrhea & 9 & 3 & 0 & 0 \\
\hline \multicolumn{5}{|l|}{ Genitourinary } \\
\hline $\begin{array}{l}\text { Frequency of urination/ } \\
\text { dysuria/urgency }\end{array}$ & 5 & 0 & 0 & 0 \\
\hline
\end{tabular}

GI, Gastrointestinal.

proposed, and radiation therapy has been frequently used to relieve severe symptoms, such as bleeding and pain, by controlling primary tumors $(6,7,13)$. Radiation therapy is very effective for local tumor control and symptom relief. However, radical radiation therapy with a curative radiation dose usually requires long overall treatment time and induces severe therapy-related toxicity in some patients. The use of radiation that achieves the minimum requirements is thus agreeable for such patients with limited survival time, although to date an optimal radiation regimen for palliative care has not been discussed in detail.

In the present study, most patients had exceedingly poor prognosis and died of disease progression. Only four and one patient survived more than 1 and 2 years after radiation therapy, respectively. Above all, all seven patients who did not undergo chemotherapy died within 1 year after radiation therapy. The use of chemotherapy was a significant variable in prolonging patient survival in this study.

The standard treatment for stage IVB uterine cervical cancer maintains systemic chemotherapy with multichemotherapeutic agents, including platinum (such as cisplatin), in many regimens $(6,7,13-15)$. For patients with stage IVB disease, chemotherapy generally cannot bring about a cure but merely a short period of prolonged survival. Furthermore, the objective response rate in such patients treated with chemotherapy is relatively low and is less than $50 \%$ at most (13-15). Recently, targeted therapy with biomolecules, such as bevacizumab, has been purposed to add further efficacy and prolong survival $(16,17)$. However, these treatments are inadequate in providing sufficient relief from severe symptoms caused by primary cervical tumors, such as bleeding and pain.

Radiation therapy is a promising treatment option for symptom relief and has a high probability of improving patients' quality of life, although there is a lack of distinct evidence that radiation therapy contributes to prolonged survival in patients with stage IVB disease. Nevertheless, since no palliative radiation regimen has been established for incurable uterine cervical cancer, familiar dose fractionations applied for palliative care of other cancer types, e.g. $30 \mathrm{~Gy}$ in 10 fractions or 20-24 Gy in 5-6 fractions, have been widely used without careful consideration. These radiation doses correspond to 28-39 $\mathrm{Gy}$ in the $\mathrm{BED}_{10}$ and 23.3-32.5 Gy in EQD2. Empirically such a radiation regimen is initially effective but is insufficient to control the cervical tumor for a longer time, and tumor regrowth is occasionally experienced in patients who survive longer than expected. Although reirradiation is considered for these patients, there is a great risk of severe toxicity, such as intestinal stenosis and perforation, because of irradiation to at-risk organs beyond their tolerable doses $(18,19)$. Therefore, planning the optimal radiation regimen according to the expected prognosis of each patient is required. However, exactly estimating the survival time of patients with varied extents of bone metastasis is exceptionally difficult. In the present study, the number of metastatic bone sites and chemotherapy were significant prognostic factors for survival. The patients who had two or fewer metastatic bone sites or received chemotherapy had an expected survival time of 1 year or longer. To the best of our knowledge, there has been no report on the relationship between the number of metastatic bone sites and survival. A more aggressive radiation regimen with a higher dose may be recommended to patients with such favorable prognostic factors, even if the overall treatment time is a little longer.

In the present study, three patients developed primary tumor progression after radiation therapy, two of whom suffered from relapse of bleeding and pain. A progressive cervical tumor greatly torments patients with such intolerable symptoms and diminishes their quality of life during their remaining period of survival. The total doses delivered to the cervical tumors and the time from irradiation to tumor progression in these three patients were $68.1,70.1$, and $82.0 \mathrm{~Gy}$ in the $\mathrm{BED}_{10}$, which corresponded to $56.8,58.4$, and 68.3 Gy in EQD2, respectively, and 7, 9, and 15 months after radiation therapy, respectively. These results suggest that patients who are expected to survive for more than 6 and 12 months may require irradiation to the cervical tumors roughly at total doses of at least 60 and 70 Gy in EQD2, respectively. In this study, most patients were treated with radiation doses higher than those generally used for palliative care, e.g. $30 \mathrm{~Gy}$ in 10 fractions or 20-24 Gy in 5-6 fractions, in order to avoid cervical tumor 
progression. Nevertheless, many of the total radiation doses used in this study were lower than the recommended dose for curative treatment. For curative radiation therapy of locally advanced cervical cancer, the American Brachytherapy Society recommends delivery of a total dose of $80 \mathrm{~Gy}$ or more in EQD2 to cervical tumors (20). By contrast, a lower radiation dose may be sufficient for patients with an expected survival time of shorter than 6 months. In the present study, the total radiation dose was not considered as a statistically significant prognostic factor for primary tumor control. This may have resulted from too short a survival time and a small number of patients.

In the present study, one patient achieved complete response to chemoradiation and survived for a relatively long time ( $\geq 5$ years). Histologically, this patient had a poorly differentiated carcinoma, such that it was impossible to make a definitive diagnosis, such as squamous cell carcinoma or adenocarcinoma. All tumors, including the primary cervical tumor and multiple metastatic lesions in the lung and bone ( $\geq 3$ metastatic bone sites), completely disappeared after treatment. The bulky primary tumor of $103 \mathrm{~mm}$ diameter was controlled by external irradiation with a total dose of 45 Gy in 25 fractions, which was considered insufficient for curative treatment of locally advanced cervical cancer. However, the remaining two patients with the same histology of poorly differentiated carcinoma died of progression of distant metastasis at 6 and 7 months after radiation therapy, although both also received aggressive chemotherapy during and following radiation therapy. Indeed, a more poorly differentiated carcinoma tends to show more rapid and invasive progression but, at the same time, in some patients, efficacy much higher than expected is observed with both chemotherapy and radiation therapy (21-23). For patients with such an uncommon histology, the treatment strategy may need to be considered independently of that for patients with a common differentiated histology, such as squamous cell carcinoma or adenocarcinoma.

In conclusion, radiation therapy is effective for the relief of severe symptoms, such as bleeding and pain, in patients with bone metastasis from uterine cervical cancer. However, some patients who developed primary tumor progression because of an insufficient radiation dose suffered from relapse of bleeding and pain. The number of metastatic bone sites and chemotherapy were significant prognostic factors for survival. For patients who are expected to survive for more than 1 year, an almost curative radiation dose should be delivered to primary cervical tumors to avoid relapse of severe symptoms and deterioration in patients' quality of life.

\section{Conflicts of Interest}

All of the Authors have declared that no actual or potential conflicts of interest exist in regard to this study.

\section{References}

1 World Health Organization. GLOBOCAN 2012: estimated incidence, mortality and prevalence worldwide in 2012. Cervical cancer. http://globocan.iarc.fr/Pages/fact_sheets_cancer.aspx. Accessed in November 27, 2017.

2 World Health Organization. Sexual and reproductive health. Comprehensive cervical cancer prevention and control - a healthier future for girls and women. WHO guidance note. http://apps.who.int/iris/bitstream/10665/78128/3/9789241505147 _eng.pdf. Accessed in November 27, 2017.

3 FIGO Committee on Gynecologic Oncology. FIGO staging for carcinoma of the vulva, cervix, and corpus uteri. Int J Gynaecol Obstet 125: 97-98, 2014.

4 Quinn MA, Benedet JL, Odicino F, Maisonneuve P, Beller U, Creasman WT, Heintz AP, Ngan HY and Pecorelli S: Carcinoma of the cervix uteri. FIGO 26th Annual Report on the Results of Treatment in Gynecological Cancer. Int J Gynaecol Obstet 95(Suppl 1): S43-S103, 2006.

5 NCCN clinical practice guidelines in oncology. Cervical cancer. Version 1. 2017. http://www.tri-kobe.org/nccn/guideline/ gynecological/english/cervical.pdf. Accessed in November 27, 2017.

6 National Cancer Institute. Cervical cancer. Cervical cancer treatment. http://www.cancer.gov/cancertopics/pdq/treatment/ cervical/HealthProfessional. Accessed in November 27, 2017.

7 Hata M, Omura M, Miyagi E, Koike I, Numazaki R, Asai-Sato M, Tayama Y, Ogino I, Hirahara F and Inoue T: The role of radiation therapy for uterine cervical cancer with distant metastasis. Oncology 83: 67-74, 2012.

8 Sobin LH, Gospodarowicz MK and Wittekind Ch. TNM Classification of Malignant Tumours. Seventh Edition. Hoboken: Wiley-Blackwell, 2010.

9 Tod MC and Meredith WJ: A dosage system for use in the treatment of cancer of the uterine cervix. Br J Radiol 11: 809824, 1938.

10 Withers HR, Thames HD Jr and Peters LJ: A new isoeffect curve for change in dose per fraction. Radiother Oncol 1: 187-191, 1983.

11 Cox JD, Stetz J and Pajak TF: Toxicity criteria of the Radiation Therapy Oncology Group (RTOG) and the European Organization for Research and Treatment of Cancer (EORTC). Int J Radiat Oncol Biol Phys 31: 1341-1346, 1995.

12 Kaplan EL and Meier P: Nonparametric estimation from incomplete observations. J Am Stat Assoc 53: 457-481, 1958.

13 Halperin EC, Wazer DE, Perez CA and Brady LW: Uterine Cervix. Principles and Practice of Radiation Oncology. (Sixth Edition). Philadelphia: Lippincott Williams \& Wilkins, 2013.

14 Long HJ 3rd: Management of metastatic cervical cancer: review of the literature. J Clin Oncol 25: 2966-2974, 2007.

15 Lorusso D, Petrelli F, Coinu A, Raspagliesi F and Barni S: A systematic review comparing cisplatin and carboplatin plus paclitaxel-based chemotherapy for recurrent or metastatic cervical cancer. Gynecol Oncol 133: 117-123, 2014.

16 Symonds RP, Gourley C, Davidson S, Carty K, McCartney E, Rai D, Banerjee S, Jackson D, Lord R, McCormack M, Hudson E, Reed N, Flubacher M, Jankowska P, Powell M, Dive C, West $\mathrm{CM}$ and Paul J: Cediranib combined with carboplatin and paclitaxel in patients with metastatic or recurrent cervical cancer (CIRCCa): a randomised, double-blind, placebo-controlled phase 2 trial. Lancet Oncol 16: 1515-1524, 2015. 
17 Rosen VM, Guerra I, McCormack M, Nogueira-Rodrigues A, Sasse A, Munk VC and Shang A: Systematic review and network meta-analysis of bevacizumab plus first-line topotecanpaclitaxel or cisplatin-paclitaxel versus non-bevacizumabcontaining therapies in persistent, recurrent, or metastatic cervical cancer. Int J Gynecol Cancer 27: 1237-1246, 2017.

18 Emami B, Lyman J, Brown A, Coia L, Goitein M, Munzenrider JE, Shank B, Solin LJ and Wesson M: Tolerance of normal tissue to therapeutic irradiation. Int J Radiat Oncol Biol Phys 21: 109-122, 1991.

19 Jones B: Toxicity after cervical cancer treatment using radiotherapy and chemotherapy. Clin Oncol 21: 56-63, 2009.

20 Viswanathan AN, Beriwal S, De Los Santos JF, Demanes DJ, Gaffney D, Hansen J, Jones E, Kirisits C, Thomadsen B and Erickson B: American Brachytherapy Society consensus guidelines for locally advanced carcinoma of the cervix. Part II: high-dose-rate brachytherapy. Brachytherapy 11: 47-52, 2012.

21 Gallup DC, Nolan TE, Hanly MG, Otken LB, Gallup DG and Maier RC: Characteristics of patients with rapidly growing cervical cancer. South Med J 90: 611-615, 1997.
22 Rose PG, Java JJ, Whitney CW, Stehman FB, Lanciano R and Thomas GM: Locally advanced adenocarcinoma and adenosquamous carcinomas of the cervix compared to squamous cell carcinomas of the cervix in Gynecologic Oncology Group trials of cisplatin-based chemoradiation. Gynecol Oncol 135: 208-212, 2014.

23 Yu JQ, Zhou Q, Zheng YF and Bao Y: Expression of vimentin and Ki-67 proteins in cervical squamous cell carcinoma and their relationships with clinicopathological features. Asian Pac J Cancer Prev 16: 4271-4275, 2015.
Received November 27, 2017

Revised December 13, 2017

Accepted December 18, 2017 